\title{
Retracing behaviors of the phase-matching angle in noncollinear phase-matched optical parametric oscillators
}

\author{
Shen-Dean Huang, Chih-Wei Hsu, Ding-Wei Huang, and C. C. Yang \\ Institute of Electro-Optical Engineering and Department of Electrical Engineering, National Taiwan University, \\ 1, Roosevelt Road, Sec. 4, Taipei, Taiwan, China
}

\begin{abstract}
We theoretically studied the retracing behaviors of the phase-matching angle in $\beta$ - $\mathrm{BaB}_{2} \mathrm{O}_{4}$ optical parametric oscillators with noncollinear phase matching when they were pumped with the harmonic of a Nd:YAG laser. The results imply that two or more sets of signal-idler outputs can be observed at certain phase-matching angles. Meanwhile, we observed that over a quite broad signal wavelength range the phase-matching condition can be reached simultaneously, leading to an optical parametric oscillator of broad signal and idler spectral widths. Experimentally, with the pump at $532 \mathrm{~nm}$, we observed a two-band signal spectrum, indicating the simultaneous phase matching of two center phase-matching angles, or retracing behavior. Also, broad signal and idler spectra were obtained with a well-chosen set of phase-matching conditions. The signal and idler spectral widths were $145 \mathrm{~nm}(745-890 \mathrm{~nm})$ and $540 \mathrm{~nm}(1.32-1.86 \mu \mathrm{m})$, respectively. (C) 1998 Optical Society of America [S0740-3224(98)01304-6]
\end{abstract}

OCIS codes: $190.0190,190.4970,190.4410$.

\section{INTRODUCTION}

Under certain phase-matching conditions, two pairs of signals and idlers can be phase matched simultaneously, and hence four output wavelengths can be obtained from an optical parametric oscillator (OPO) if the two signals oscillate in the cavity. This phenomenon is called the retracing behavior of the phase-matching angle (RBPA), ${ }^{1-3}$ which means that the phase-matched signal wavelength is not a monotonically increasing or decreasing function of crystal orientation angle. RBPA was first observed by Lin et $a l^{1,2}$ in the second-harmonic generation (SHG) from a lithium triborate (LBO) crystal. They found that two different fundamental wavelengths of SHG could have the same phase-matching angle in LBO. The RBPA in an OPO with collinear phase matching was first studied by Liu et al. ${ }^{3}$ in many other crystals, such as $\beta-\mathrm{BaB}_{2} \mathrm{O}_{4}$ (BBO), $\mathrm{LiIO}_{3}, \mathrm{GaSe}, \mathrm{KH}_{2} \mathrm{PO}_{4}, \mathrm{AgGaS}_{2}$, and $\mathrm{AgGaSe}_{2}$. They showed theoretically that when the pump wavelength was in some specific range, the RBPA could be observed. In Table 1 we list the pump wavelength ranges required for observing the RBPA with type I phase matching and the transparency ranges ${ }^{4}$ for 10 nonlinear crystals. In this table we summarize the calculated results of Ref. 3 and ours. Experimentally, a four-wavelength $\mathrm{OPO}^{5}$ and optical parametric amplifiers $^{6-9}$ were indeed demonstrated with LBO crystals pumped with 523- and 532-nm lasers. However, under collinear phase-matching conditions the implementation of a four-wavelength OPO is still hindered by the limited ranges of pumping wavelength. For example, BBO shows RBPA only when the pump wavelength falls into the range between 560 and $680 \mathrm{~nm}$, which is not covered by commonly used high-power lasers, such as the harmonic of Nd:YAG lasers or XeCl excimer lasers. Except for LBO, most other crystals have the same limitation as BBO, as can be seen from Table 1 .
In this paper we propose that in noncollinear phasematching configurations it is possible to realize retracing phenomena with the pump wavelengths falling into the wavelength ranges of available high-power laser systems. In particular, our calculations have predicted that fourwavelength OPO's can be realized in BBO with pump wavelengths at 532, 355, and $266 \mathrm{~nm}$ in noncollinear phase-matching configurations. Also, we found that, near certain phase-matching angles, phase matching could be simultaneously achieved over quite a broad spectral width. Experimentally we observed a retracing phenomenon in a BBO OPO pumped with the second harmonic of a Nd:YAG laser. Meanwhile, near the turning point of the phase-matching curve, we implemented an OPO with broad signal and idler spectral widths (145 nm in the signal and $540 \mathrm{~nm}$ in the idler). With certain simple tuning mechanisms, such a coherent light source of broad spectral width should find many applications in spectroscopic measurements and others. Recently Wang et al. demonstrated a broad signal spectrum from a BBO OPO pumped with a $355-\mathrm{nm}$ signal. ${ }^{10}$ Although they also predicted the possibility of observing similar broadsignal spectra in other nonlinear crystals with other pump wavelengths, their discussions were not complete. In this paper we present a more comprehensive description of the broad-spectrum phenomenon. In particular, for what we believe is for the first time, we include a detailed discussion of the retracing behaviors of noncollinear phase-matched OPO's with various nonlinear crystals. We present our theoretical analysis in Section 2 and then our experimental results in Section 3. Conclusions are drawn in Section 4.

\section{THEORETICAL ANALYSIS}

In this section we present our theoretical analysis and numerical results of the RBPA. Most of our results are 
based on BBO crystal, which is one of the most important OPO crystals. Both type I and type II phase-matching conditions are considered. Figure 1 shows the orientations of various waves in an OPO with the noncollinear phase-matching configuration. $\quad \mathbf{K}_{p}$ is the wave vector of the pump beam, which lies at a phase-matching angle $\theta$ from the optic axis. The Poynting vector of the pump beam is represented by $\mathbf{P}_{p}$, which lies at a walk-off angle $\rho$ with respect to $\mathbf{K}_{p} . \quad \mathbf{K}_{s}$ and $\mathbf{K}_{i}$, which are the wave vectors of the signal and the idler, lie at angles $\delta$ and $\gamma$ with respect to $\mathbf{K}_{p}$, respectively. Note that when $\delta$ $=0$, a collinear phase-matching configuration results. For the noncollinear phase-matching shown in Fig. 1 the phase-matching condition is satisfied with

$$
\begin{aligned}
\sin (\delta) n_{o}\left(\lambda_{s}\right) / \lambda_{s} & =\sin (\gamma) n_{o}\left(\lambda_{i}\right) / \lambda_{i} \\
\cos (\delta) n_{o}\left(\lambda_{s}\right) / \lambda_{s}+ & \cos (\gamma) n_{o}\left(\lambda_{i}\right) / \lambda_{i} \\
& =\tilde{n}_{e}\left(\theta, \lambda_{p}\right) / \lambda_{p} \\
1 / \tilde{n}_{e}{ }^{2}\left(\theta, \lambda_{p}\right) & =\cos ^{2} \theta / n_{o}^{2}\left(\lambda_{p}\right)+\sin ^{2} \theta / n_{e}{ }^{2}\left(\lambda_{p}\right) .
\end{aligned}
$$

Here $\lambda_{p}, \lambda_{s}$, and $\lambda_{i}$ are the wavelengths of the pump, the signal, and the idler, respectively. Also, $n_{o}$ and $n_{e}$ are the two refractive indices of the uniaxial crystal, as usually defined; $\tilde{n}_{e}$ is the refractive index of the extraordinary wave, which is a function of the polar angle $\theta$ be-

Table 1. Pump Wavelength Required for RBPA $\left(\lambda_{R}\right)$ in Various Type I Collinear Phase-Matched Nonlinear Optical Crystals and Their Transparency Ranges

\begin{tabular}{lcc}
\hline Crystal & Transparency range $(\mu \mathrm{m})^{a}$ & $\lambda_{R}(\mu \mathrm{m})$ \\
\hline $\mathrm{BBO}$ & $0.19-3.5$ & $0.56-0.68^{b}$ \\
$\mathrm{KDP}$ & $0.20-1.5$ & $0.44-0.48^{b}$ \\
$\mathrm{ADP}$ & $0.20-1.2$ & $0.45-0.50^{b}$ \\
$\mathrm{AgGaS}$ & $0.50-13$ & $0.60-0.85^{b}$ \\
$\mathrm{AgGaSe}_{2}$ & $0.71-18$ & $0.65-0.73^{b}$ \\
$\mathrm{LiIO}_{3}$ & $0.30-5.5$ & $0.75-1.05^{b}$ \\
$\mathrm{GaSe}_{\mathrm{LBO}}$ & $0.65-18$ & $2.0-2.9^{b}$ \\
$\mathrm{LiNbO}_{3}$ & $0.16-2.6$ & $0.52-0.59(X Y$ plane $)$ \\
$\mathrm{KNbO}_{3}$ & $0.33-5.5$ & $0.78-0.93$ \\
\hline
\end{tabular}

${ }^{a}$ Ref. 4.

${ }^{b}$ Ref. 3.

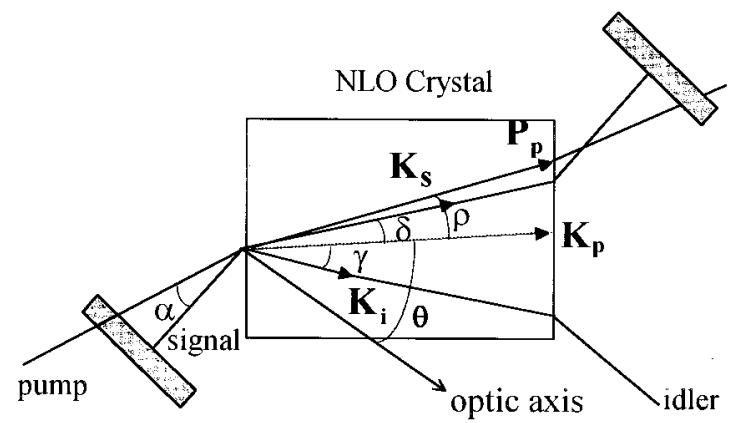

Fig. 1. Noncollinear phase-matching configuration for type I phase matching in a negative crystal. NLO, nonlinear optical.
Table 2. Range of the $\delta$ Angle for RBPA with

Type I Noncollinear Phase Matching in Four Crystals Pumped with the Harmonics of a Nd:YAG Laser and a 308-nm XeCl Laser ${ }^{a}$

\begin{tabular}{lccc}
\hline \multicolumn{1}{c}{ Crystal } & $\lambda_{p}(\mathrm{~nm})$ & $\delta(\mathrm{deg})$ & $\rho(\mathrm{deg})$ \\
\hline BBO & 266 & $2.8-6.0$ & $\sim 4.5$ \\
& 308 & $2.5-5.0$ & $\sim 4.5$ \\
& 355 & $2.3-4.3$ & $\sim 4.2$ \\
$\mathrm{LBO}$ & 532 & $1.0-2.3$ & $\sim 3.3$ \\
& 308 & $2.7-4.3$ & $1.6-1$ \\
$\mathrm{LiNbO}_{3}$ & 355 & $2.4-3.5$ & $\sim 1.6$ \\
$\mathrm{KNbO}_{3}$ & 532 & $0.0-1.3$ & $\sim 0.5$ \\
& 532 & $3.0-4.0$ & $1.5-0$ \\
\hline
\end{tabular}

${ }^{a}$ The approximate $\rho$ angles are also shown.

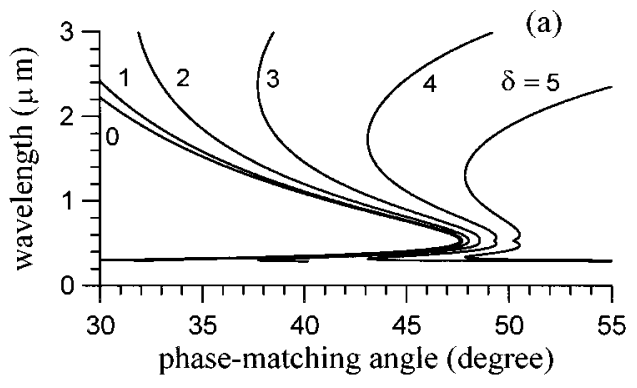

(b)

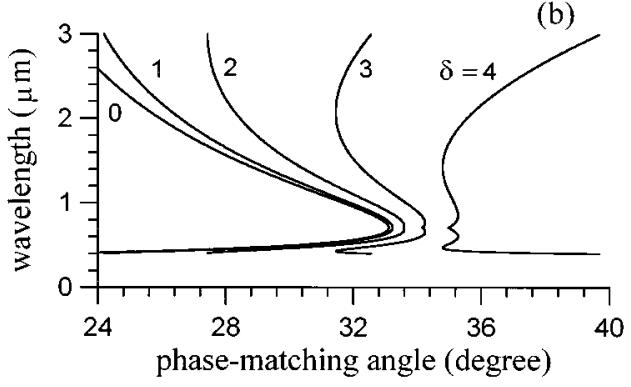

(c)

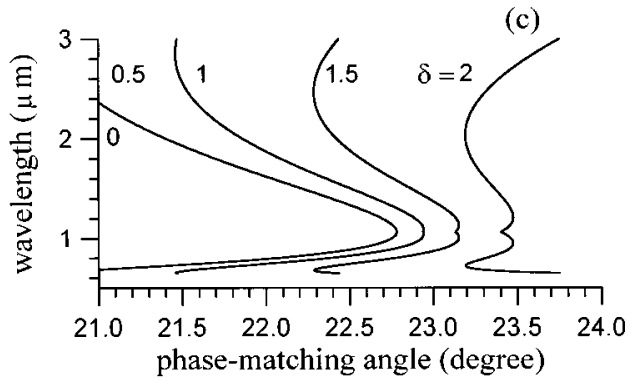

Fig. 2. Tuning curves in a type I phase-matched BBO OPO with (a) $\lambda_{P}=266 \mathrm{~nm}$, (b) $\lambda_{P}=355 \mathrm{~nm}$, (c) $\lambda_{P}=532 \mathrm{~nm}$. The numbers shown next to the curves are the corresponding $\delta$ angles.

tween the $z$ axis and the wave vector. Equations (1)-(3) are solved for the following results. Their values can be obtained from the Sellmier equations. ${ }^{4}$

In Table 2 we list the calculated $\delta$ values and the corresponding $\rho$ values for the RBPA in four commonly used OPO crystals with a pump wavelength $\lambda_{P}$ at the second $(532 \mathrm{~nm})$, third $(355 \mathrm{~nm})$, and fourth $(266 \mathrm{~nm})$ harmonics of a Nd:YAG laser and of a $\mathrm{XeCl}(308 \mathrm{~nm})$ excimer laser. It should be noted that when $\rho$ equals $\delta, \mathbf{K}_{s}$ and $\mathbf{P}_{p}$ over- 
lap, resulting in the lowest oscillation threshold of the OPO. ${ }^{11}$ It can be seen from Table 2 that all the four crystals show RBPA with the available pumping wavelengths when suitable noncollinear phase-matching configurations are applied. Furthermore, we notice that some crystals, such as BBO pumped by a 266-, a 308-, or a $355-n m$ laser, show RBPA with the $\delta$ angle approximately equal to the $\rho$ angle, leading to the lowest oscillation threshold. Figure 2 shows the tuning curves of the BBO OPO pumped with 266-, 355-, and 532-nm lasers at various $\delta$ angles. When $\delta$ increases, the phase-matching angle $\theta$ shifts to a larger value. We see that when $\delta$ exceeds a certain value, the RBPA occurs. Besides, at some $\delta$ angles, even three pairs of signals and idlers can be phase matched simultaneously. This phenomenon implies that the implementation of a six-wavelength OPO might be possible if ultrabroadband mirrors were available.

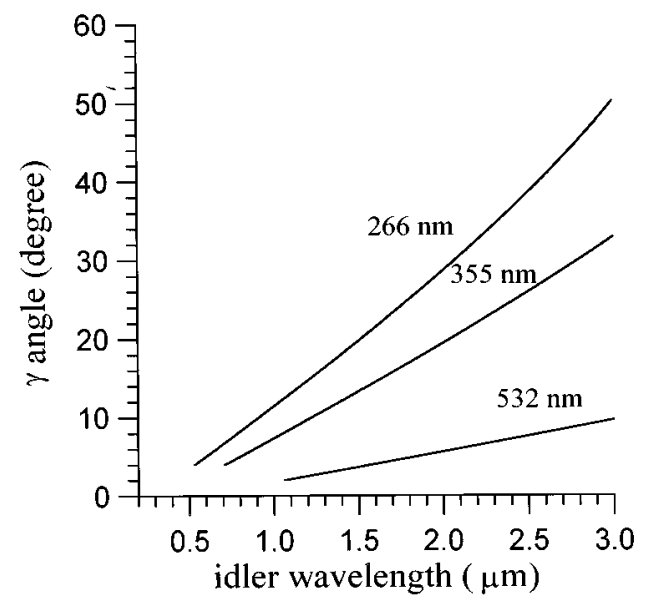

Fig. 3. $\quad \gamma$ angle versus idler wavelength $\lambda_{i}$ in a type I phasematched BBO OPO with $\lambda_{P}=266,355,532 \mathrm{~nm}$. The corresponding $\delta$ angles are $4^{\circ}, 4^{\circ}$, and $2^{\circ}$, respectively.

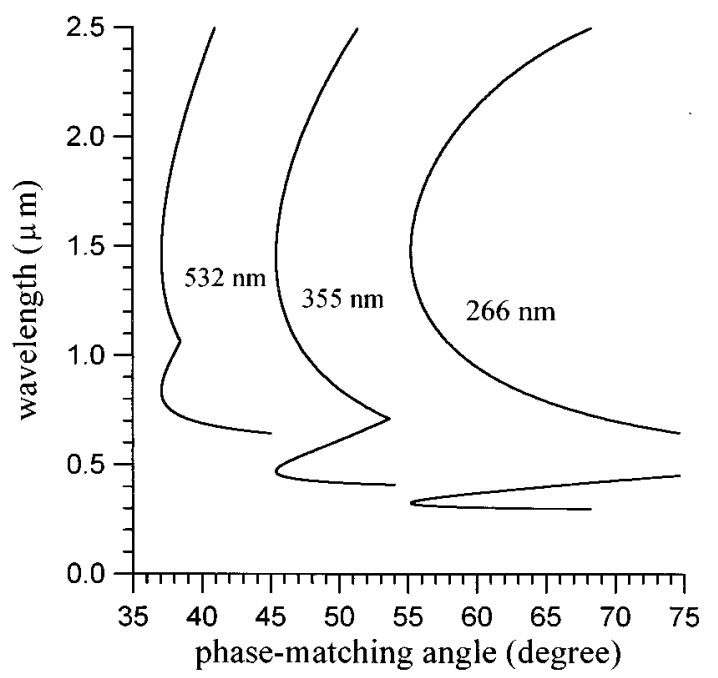

Fig. 4. Tuning curves in a type II phase-matched BBO OPO at $\delta=5^{\circ}$, with $\lambda_{P}=266,355,532 \mathrm{~nm}$.

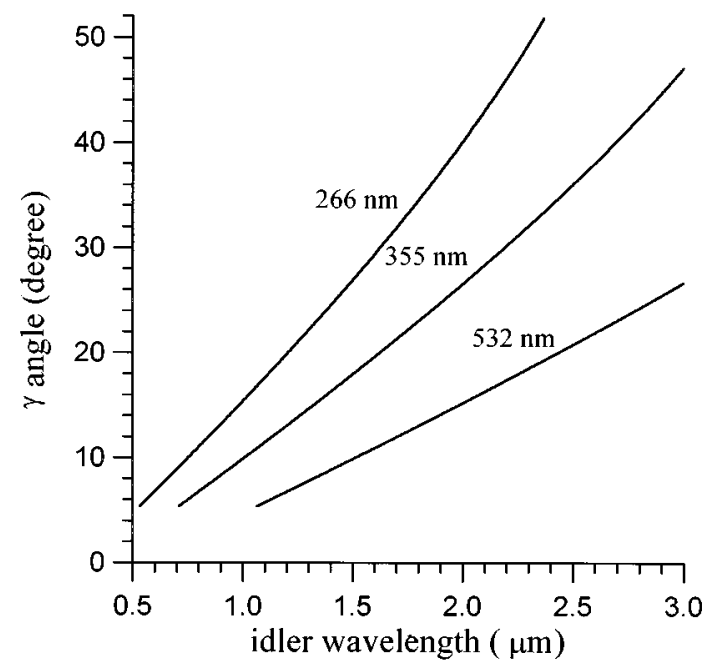

Fig. 5. $\quad \gamma$ angle versus idler wavelength $\lambda_{i}$ in a type II phasematched BBO OPO at $\delta=5^{\circ}$, with $\lambda_{P}=266,355,532 \mathrm{~nm}$.

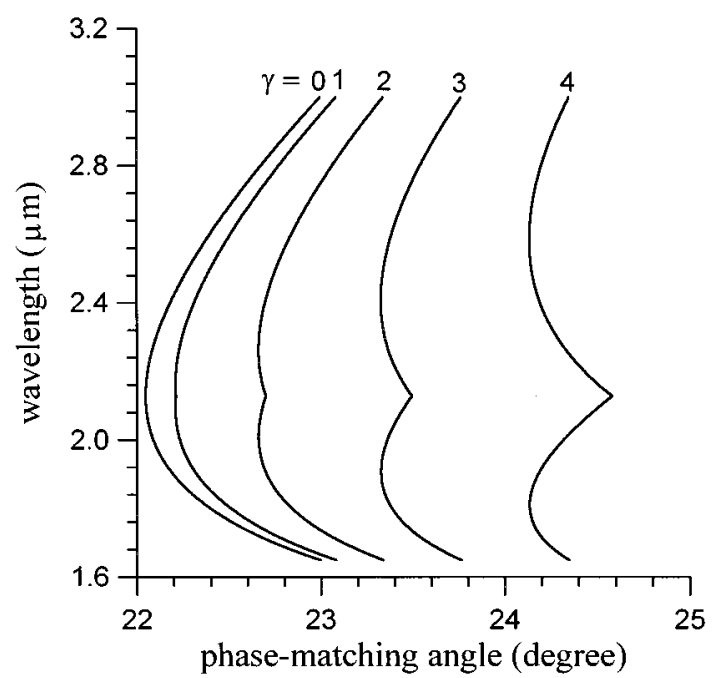

Fig. 6. Tuning curves in a type I phase-matched BBO OPO with $\lambda_{P}=1064 \mathrm{~nm}$. The numbers shown above the curves are the corresponding $\gamma$ angles.

A possible disadvantage of an OPO based on noncollinear phase matching is that when $\delta$ increases, $\gamma$ increases fast, particularly when the signal wavelengths are far from degeneracy. In Fig. 3 we show the $\gamma$ angle versus the idler wavelength $\lambda_{i}$ of BBO OPO with pump wavelength $\lambda_{P}$ at 266, 355, and $532 \mathrm{~nm}$. The $\delta$ angles for the three curves are $4^{\circ}, 4^{\circ}$, and $2^{\circ}$, respectively. The large $\gamma$ angle implies that one should use a crystal of a large transverse size to collect the idler beam. Meanwhile, a large $\gamma$ angle results in a lower conversion efficiency. In addition, we notice that the idler beam direction varies with wavelength, which makes the collection of idler output difficult. To show that RBPA with noncollinear phase matching also exists in type II phase matching we plot the tuning curves of a type II phase-matched BBO OPO in Fig. 4. The three curves in this figure correspond to pump wavelengths at 266,355 , and $532 \mathrm{~nm}$, all with $\delta=5^{\circ}$. Figure 5 shows the dependencies of 
idler beam directions on wavelength, i.e., $\gamma$ versus $\lambda_{i}$, in the cases of Fig. 4.

All the results described above refer to cases in which $\delta$ angles are fixed while the output wavelengths are tuned. According to our definitions, the physical meaning of fixed $\delta$ is that the short-wavelength component in the parametric process (called the signal) is resonated in an OPO, because the direction of the signal beam is determined by the resonator mirrors. On the other hand, one can resonate the long-wavelength component (which will still be called the idler) instead of the signal. In such a case the $\gamma$ angle instead of the $\delta$ angle is fixed. We can also observe RBPA in the cases in which the idler is resonated. Figure 6 shows the tuning curves of a type I phasematched BBO OPO pumped with a 1064-nm laser at various $\gamma$ angles. From our numerical results we find that when the signals are resonated, RBPA occurs only when pump wavelength $\lambda_{P}$ is shorter than $\lambda_{R}$ (the wavelength required for RBPA in collinear phase matching, as shown in Table 1). However, for $\lambda_{P}$ longer than $\lambda_{R}$, RBPA in noncollinear phase matching occurs only when the idlers are resonated. This phenomenon was observed in all the crystals that we calculated, including $\mathrm{BBO}, \mathrm{LBO}, \mathrm{KNbO}_{3}$, and $\mathrm{LiNbO}_{3}$.

From the results above we see that at some specific $\delta$ angle the phase-matching spectral width can be very large, typically more than $100 \mathrm{~nm}$. For example, in Fig. 2(c) the curve with $\delta=1.5^{\circ}$ shows such a feature near $\theta=23.1^{\circ}$. In this situation an OPO of broad spectral width can be implemented. If dispersive components, such as gratings, are introduced into the cavity, ${ }^{12,13}$ or if tunable injection seeding is applied, ${ }^{14-16}$ we can tune the OPO with the narrow linewidth throughout the whole wavelength range without rotating the crystal. It is anticipated that this kind of device should be useful for implementing a master oscillator-power oscillator setup. $^{17}$

\section{EXPERIMENTS AND RESULTS}

In this section we present the experimental procedure and RBPA results of a BBO OPO pumped with the second harmonic $(532 \mathrm{~nm})$ of a $Q$-switched Nd:YAG laser. Figure 7 shows the cavity configuration of our noncollinear phase-matched OPO. The OPO cavity consisted of two plane mirrors (M1 and M2), which were coated centered at $810 \mathrm{~nm}$ with reflectivities of $99.5 \%$ and $98 \%$ (output coupler), respectively. We used a $2 \times$ telescope (the combination of lenses L1 and L2) to reduce the pump-beam diameter to $2.75 \mathrm{~mm}$. The cavity length was $2.5 \mathrm{~cm}$. The pump source was the second harmonic of a singlelongitudinal-mode $Q$-switched Nd:YAG laser (Coherent Infinity 40-100). The pulsed laser, with 5-ns duration and a top-hat spatial profile, was operated at a repetition rate of $10 \mathrm{~Hz}$. The oscillation threshold for the OPO was $\sim 12 \mathrm{~mJ} /$ pulse. Our BBO crystal with dimensions $10 \mathrm{~mm}$ $\times 8 \mathrm{~mm} \times 7 \mathrm{~mm}$ was cut for type I phase matching $(\theta$ $\left.=20^{\circ}, \phi=0^{\circ}\right)$. Its facet was antireflection coated centered at $810 \mathrm{~nm}$.

Figure 8 shows a closeup of the theoretical tuning curve of $\mathrm{BBO}$ with $\alpha=3.7^{\circ}-4.2^{\circ}\left(\delta=2.2^{\circ}-2.49^{\circ}\right)$ with $532-\mathrm{nm}$ pumping. Here, $\alpha$ is the angle between the

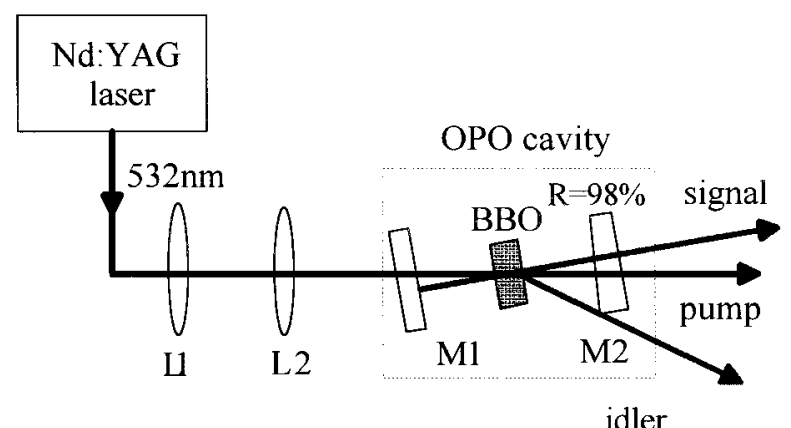

Fig. 7. Schematic cavity configuration of the noncollinear phase-matched BBO OPO.

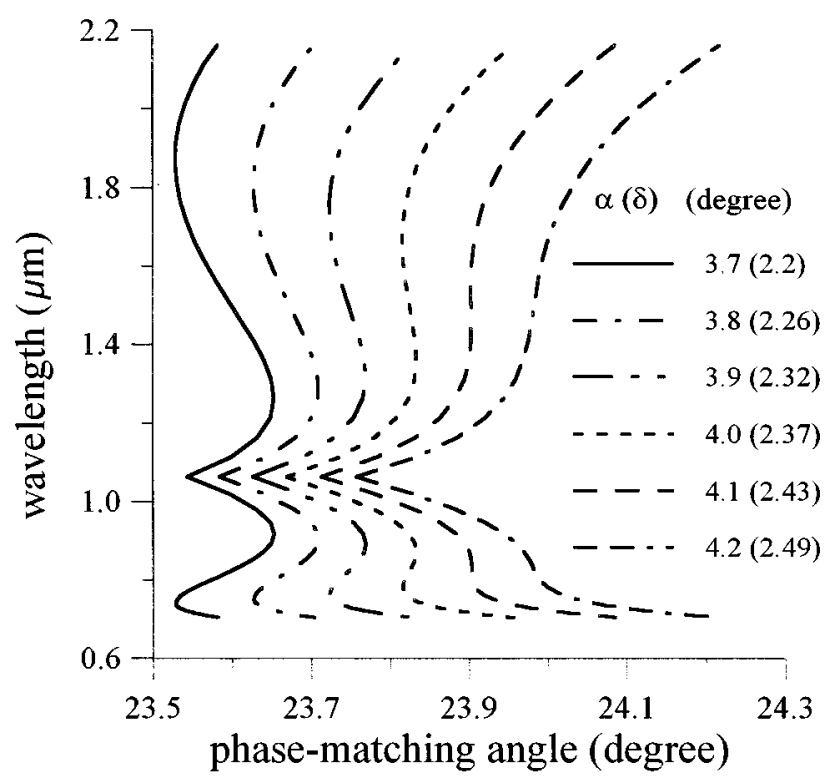

Fig. 8. Theoretical tuning curves pumped with a 532-nm laser. The BBO crystal was cut at $\theta=20^{\circ}$. Note that $\alpha$ is defined as the angle between the pump and signal beams in the air.

pump and the signal in the air, as shown in Fig. 1. The RBPA is clearly seen for $\alpha$ in this range. The optimum high-reflection coating range of the cavity mirrors for studying the RBPA is approximately $700-800$ or $850-950$ $\mathrm{nm}$. The coatings of our cavity mirrors and crystal are not optimized. Hence, it is difficult to follow experimentally the whole trace of the RBPA. Nevertheless, around the coating wavelength we could still observe the simultaneous oscillation of two signal-idler sets. Figure 9 shows the spectrum of the two bands of the signal wavelength. The results were obtained at $\alpha=3.9^{\circ} \quad(\delta$ $=2.32^{\circ}$ ) and $20-\mathrm{mJ} /$ pulse pump energy. According to our theoretical predictions, the peaks of the two bands should be at 728 and $894 \mathrm{~nm}$. However, the coating range limits the observation of the whole spectrum.

From Fig. 8 we can observe that, when $\alpha$ is close to $4.1^{\circ}$ ( $\delta$ is close to $2.43^{\circ}$ ), the tuning curve becomes almost vertical in the signal wavelength range from 800 to $900 \mathrm{~nm}$. This means that we can obtain a broad-signal spectral width from the phase-matched OPO if $\theta$ and $\alpha$ are appropriately chosen. Figure 10 shows the experimental results when $\alpha$ is close to $4.25^{\circ}$ ( $\delta$ is close to $2.52^{\circ}$ ) and the 
pump energy is $30 \mathrm{~mJ} /$ pulse. Here we can see that the spectral width ranges from 745 to $890 \mathrm{~nm}$. The corresponding idler wavelength range is from 1.32 to $1.86 \mu \mathrm{m}$. The spectral widths of the signal and idler are as large as 145 and $540 \mathrm{~nm}$, respectively. These spectral widths are actually limited by the cavity mirror coating. Even broader spectral widths could be expected if the coating range were optimized. If we use a tuning setup, such as

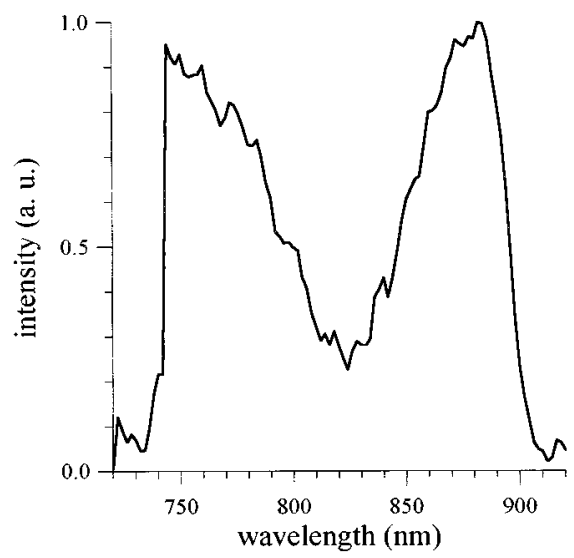

Fig. 9. Signal spectrum of the OPO. The two separate bands indicate the RBPA.

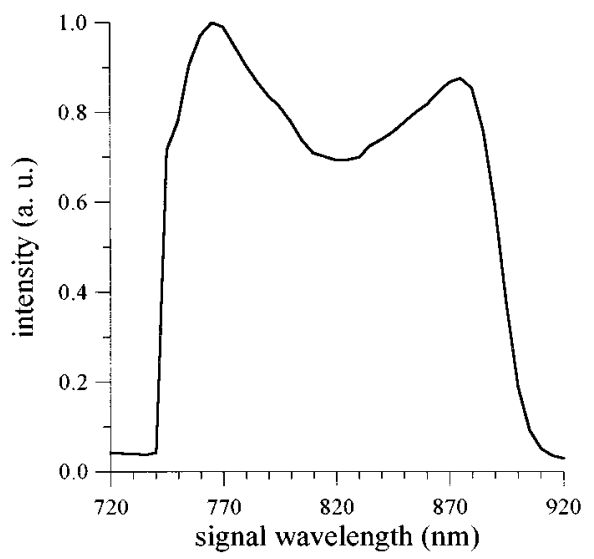

Fig. 10. Signal spectrum of the OPO of a phase-matching case of broad spectral width.

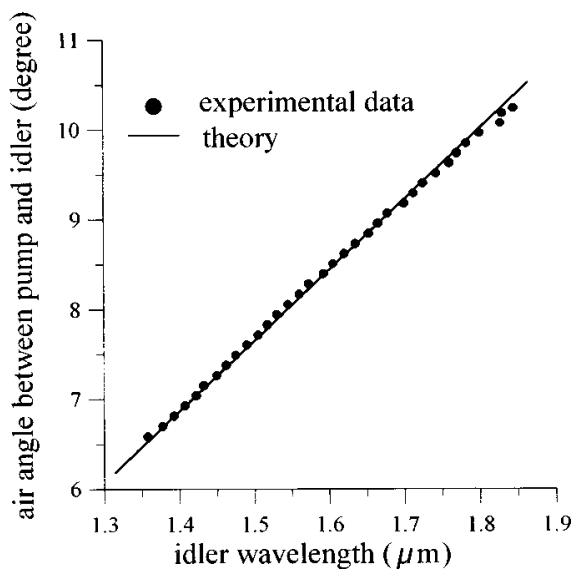

Fig. 11. Experimental results and theoretical predictions for the air angle between the pump and the idler as a function of the idler wavelength.

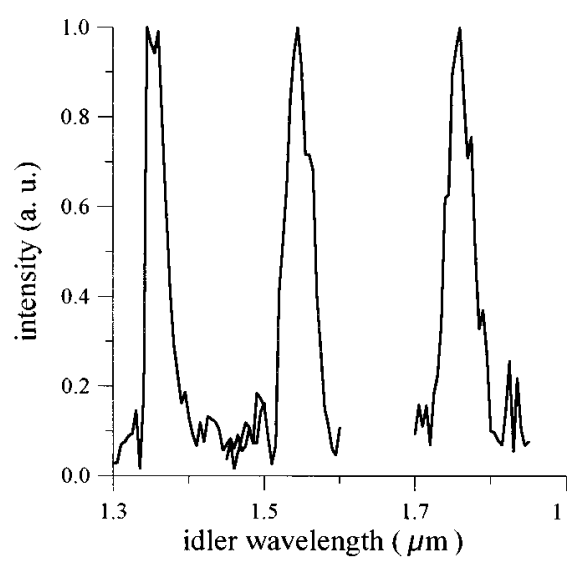

Fig. 12. Three idler spectra, corresponding to the signal in Fig. 10.

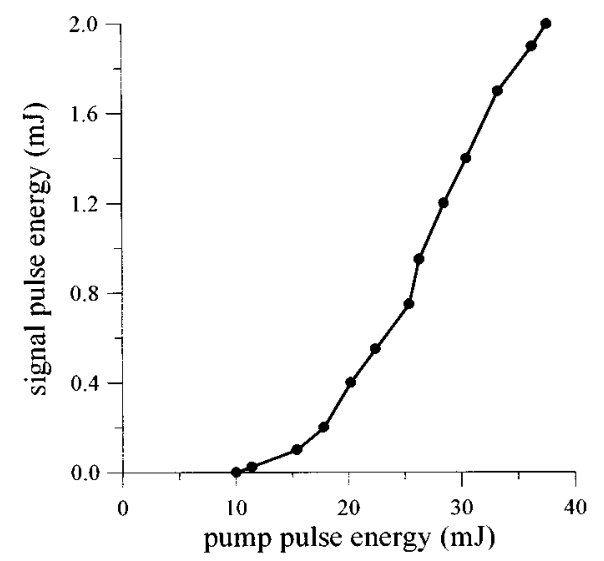

Fig. 13. Output pulse energy under the phase-matching condition of the broad spectral width as a function of the pump-pulse energy.

an intracavity grating, we can obtain broadly tunable coherent sources in the wavelength ranges given above without complicated tuning arrangements.

As we mentioned above, a drawback of such a system is that the idler spreads in space for several degrees. Therefore it is difficult to collect the whole idler beam with a simple setup. However, with a collecting fiber of small aperture, which is mounted upon a translation stage, it is possible to obtain a source that is tunable from 1.32 to $1.86 \mu \mathrm{m}$. In Fig. 11 we show the experimental result of the air angle between the pump and the idler for the case of Fig. 10. It can be seen that the spreading angle of the idler is $\sim 3.7^{\circ}$. This result is consistent with our theoretical calculation (the solid curve in the same figure). Figure 12 shows three idler spectra obtained when we moved the collecting fiber bundle (with a diameter of $1.4 \mathrm{~mm}$ ) along the translation stage. The FWHM of each idler spectrum is approximately $30-40 \mathrm{~nm}$.

In Fig. 13 we show the signal-pulse energy as a function of the pump-pulse energy. At the maximum pump energy $(37.6 \mathrm{~mJ})$, the conversion efficiency into the signal is $5.3 \%$. This value is somewhat lower than those previously reported. ${ }^{11}$ The efficiency that we obtained resulted mainly because we use an output coupler with high reflectivity (98\%). After a few round trips, the signal en- 
ergy within the cavity was saturated. Besides, the antireflection coating of the $\mathrm{BBO}$ crystal and the first cavity mirror (M1) reflect a pump-beam energy as high as $35 \%$. We expect that with an output coupler of lower reflectivity and appropriate crystal coating, OPO's of either fourwavelength or broad spectral widths can be implemented with higher conversion efficiencies.

\section{CONCLUSIONS}

We have shown theoretically and experimentally the retracing behaviors of the phase-matching angle in $\mathrm{BBO}$ OPO's with noncollinear phase matching (either type I or type II). Theoretically, the RBPA in BBO OPO's could be observed when the OPO's were pumped by the fourth, third, and second harmonics of a Nd:YAG laser and by itself. These phenomena were more prominent with a shorter pump wavelength. We noticed that near some specific phase-matching angles there existed broad-signal wavelength ranges over which the phase-matching condition could be simultaneously reached. In experiments we implemented a BBO OPO pumped with the second harmonic of a $Q$-switched Nd:YAG laser. Although the coatings of the cavity mirrors and the BBO crystal were not optimized, we could still observe two bands of signal, indicating the generation of two signal-idler sets. Meanwhile, we obtained broad signal and idler spectral widths with a set of well-chosen phase-matching angles. The signal and idler spectra range from 745 to $890 \mathrm{~nm}$ and from 1.32 to $1.86 \mu \mathrm{m}$, respectively.

\section{ACKNOWLEDGMENT}

This research was sponsored by grants NSC 86-2215-E002-010 and NSC 86-2215-E-002-011 from the National Science Council, The Republic of China.

\section{REFERENCES}

1. S. Lin, B. Wu, F. Xie, and C. Chen, "Phase-matching retracing behavior: new features in $\mathrm{LiB}_{3} \mathrm{O}_{5}$," Appl. Phys. Lett. 59, 1541-1543 (1991).

2. S. Lin, B. Wu, F. Xie, and C. Chen, "Phase-matching retracing behavior for second harmonic generation in $\mathrm{LiB}_{3} \mathrm{O}_{5}$ crystal," J. Appl. Phys. 73, 1029-1034 (1993).

3. X. Liu, D. Deng, M. Li, D. Guo, and Z. Xu, "Retracing behavior of the phase-matching angle of nonlinear crystals in optical parametric oscillators," J. Appl. Phys. 74, 2989 2991 (1993).
4. V. G. Dmitriev, G. G. Gurzdyan, and D. N. Nikogosyan, Handbook of Nonlinear Optical Crystals (Springer-Verlag, Berlin, 1991).

5. G. J. Hall, M. Ebrahimzadeh, A. Robertson, G. P. A. Malcolm, and A. I. Ferguson, "Synchronously pumped optical parametric oscillators using all-solid-state pump lasers," J. Opt. Soc. Am. B 10, 2168-2179 (1993).

6. S. Lin, J. Y. Huang, J. Ling, C. Chen, and Y. R. Shen, "Optical parametric amplification in a lithium triborate crystal tunable from 0.65 to $2.5 \mu \mathrm{m}$," Appl. Phys. Lett. 59, 28052807 (1991).

7. J. Y. Zhang, J. Y. Huang, Y. R. Sheng, and C. Chen, "Optical parametric generation and amplification in barium borate and lithium triborate crystals," J. Opt. Soc. Am. B 10, 1758-1764 (1993).

8. Z. Xu, X. Liu, D. Deng, Q. Wu, L. Wu, B. Wu, S. Lin, B. Lin, and C. Chen, "Multiwavelength optical parametric amplification with angle-tuned lithium triborate," J. Opt. Soc. Am. B 12, 2222-2228 (1995).

9. X. Liu, Z. Xu, B. Wu, and C. Chen, "Picosecond multiwavelength optical parametric generation in lithium triborate," Appl. Phys. Lett. 66, 1446-1448 (1995).

10. J. Wang, M. H. Dunn, and C. F. Rae, "Polychromatic optical parametric generation by simultaneous phase matching over a large spectral bandwidth," Opt. Lett. 22, 763-765 (1997).

11. L. A. W. Gloster, Z. X. Jiang, and T. A. King, "Characterization of an Nd:YAG-pumped $\beta-\mathrm{BaB}_{2} \mathrm{O}$ optical parametric oscillator in collinear and noncollinear phase-matched configurations," IEEE J. Quantum Electron. 30, 2961-2969 (1994).

12. L. A. W. Gloster, I. T. McKinnie, Z. X. Jiang, T. A. King, J. M. Boon-Engering, W. E. van der Veer, and W. Hogervorst, "Narrow-band $\beta-\mathrm{BaB}_{2} \mathrm{O}_{4}$ optical parametric oscillator in a grazing-incidence configuration," J. Opt. Soc. Am. B 12, 2117-2121 (1995).

13. B. C. Johnson, V. J. Newell, J. B. Clark, and E. S. McPhee, "Narrow-bandwidth low-divergence optical parametric oscillator for nonlinear frequency-conversion applications," J. Opt. Soc. Am. B 12, 2122-2127 (1995).

14. A. Fix, T. Schroder, R. Wallenstein, J. G. Haub, M. J. Johnson, and B. J. Orr, "Tunable $\beta$-barium borate optical parametric oscillator: operating characteristics with and without injection seeding," J. Opt. Soc. Am. B 10, 17441750 (1993).

15. J. G. Haub, R. M. Hentschel, M. J. Johnson, and B. J. Orr, "Controlling the performance of a pulsed optical parametric oscillator: a survey of techniques and spectroscopic applications," J. Opt. Soc. Am. B 12, 2128-2141 (1995).

16. A. Fix and R. Wallenstein, "Spectral properties of pulsed nanosecond optical parametric oscillators: experimental investigation and numerical analysis," J. Opt. Soc. Am. B 13, 2484-2497 (1996).

17. B. C. Johnson, V. J. Newell, J. B. Clark, and E. S. McPhee, "Narrow-bandwidth low-divergence optical parametric oscillator for nonlinear frequency-conversion applications," J. Opt. Soc. Am. B 12, 2122-2127 (1995). 